\title{
Transition from In Situ to Invasive Testicular Germ Cell Neoplasia is Associated with the Loss of p21 and Gain of $\mathbf{m d m}-2$ Expression
}

\author{
Milton W. Datta, Ettore Macri, Sabina Signoretti, Andrew A. Renshaw, Massimo Loda \\ Departments of Pathology, Medical College of Wisconsin (MWD), Milwaukee, Wisconsin, Baptist Hospital \\ of Miami (AAR), Miami, Florida, and Brigham and Women's Hospital (ML), Boston, Massachusetts, and \\ Division of Tumor Biology (EM, SS, ML), Dana Farber Cancer Institute, Boston, Massachusetts
}

Introduction: The tumor suppressor gene p53 has been shown to transcriptionally regulate expression of the cell cycle dependent kinase inhibitor p21. p53 is in turn regulated by the ubiquitin ligase mouse double minute-2 (mdm-2). We have set out to examine p21 expression in testicular germ cell tumors and its relationship with p53 and mdm-2 expression. Methods: Immunohistochemical analysis was performed on formalin-fixed paraffin-embedded tissue for p53, p21, and mdm-2 in 31 testicular germ cell tumors, which included 17 pure seminomas and 14 mixed germ cell tumors composed predominantly of embryonal carcinoma. Twenty-seven cases contained adjacent areas of intratubular germ cell neoplasia (ITGCN). Results: 17 out of 17 seminomas and 14 out of 14 embryonal carcinomas expressed p53 in both ITGCN and the invasive tumor. In contrast, none of the 17 seminomas and only 2 of 14 embryonal carcinomas revealed positive staining for p21 protein. p21 expression was noted in 18 of 27 cases $(67 \%)$ of ITGCN, and in 16 of these cases $(89 \%)$ the corresponding invasive tumor had lost p21 expression. In nine additional cases p21 expression was absent in both the invasive and intratubular tumor. mdm-2 expression was present in 8 out of $17(47 \%)$ seminomas and 13 out of $14(93 \%)$ embryonal carcinomas but was present in only 2 out of $27(7 \%)$ cases of ITGCN. Statistically significant associations for loss of p21 and gain of mdm-2 expression in invasive tumors were present $(P<$ .0001). Conclusions: The co-expression of p53 and p21 in ITGCN is consistent with preservation of p53-

Copyright (C) 2001 by The United States and Canadian Academy of Pathology, Inc.

VOL. 14, NO. 5, P. 437, 2001 Printed in the U.S.A.

Date of acceptance: August 2, 2000.

Presented in part at the 89th annual meeting of the United States and Canadian Academy of Pathology, New Orleans, March 25-31, 2000.

Address reprint requests to: Milton W. Datta, Department of Pathology, Froedtert Memorial Lutheran Hospital, Medical College of Wisconsin, 9200 W. Wisconsin Ave., Milwaukee, WI 53226; e-mail: mdatta@mcw.edu; fax: 414-805-8444. directed induction of p21. The loss of p21 expression in invasive tumors suggests a disruption of the p53 regulatory pathway. The inverse correlation of p21 and mdm-2 expression in both ITGCN and invasive tumors could indicate that loss of the functional p53 regulatory pathway may be correlated with the onset of mdm-2 expression. These results raise the possibility that the loss of p21 expression may be associated with the development of invasive germ cell tumors from ITGCN. Persistent p53 expression in the presence of $\mathbf{m d m}-2$ suggests that in testicular germ cell tumors, while mdm-2 can block the transactivation potential of p53, it can no longer target p53 for degradation.

KEY WORDS: Testis, Germ cell tumors, p53, p21, mdm-2, Intratubular germ cell neoplasia.

Mod Pathol 2001;14(5):437-442

Testicular germ cell tumors express unusually high levels of p53, whereas mutational analyses have shown that the p53 expressed is wild type (1-3). p53 induces the expression of p21, an inhibitor of the cyclin/cyclin-dependent kinase complexes involved in control of the cell cycle (4). The loss of p21 expression can lead to augmented cell proliferation, increased risk of mutational events, and oncogenesis. A major regulator of p53 is the ubiquitin ligase mouse double minute-2 (mdm-2), which is induced by p53 and in turn binds and functionally inactivates p53 protein by targeting it for degradation (5). In this paper we have examined this p53 mediated regulatory pathway in 31 testicular germ cell tumors (17 seminomas and 14 embryonal carcinomas) as well as their in situ component, intratubular germ cell neoplasia (ITGCN) through the associated expression of p53, p21, and mdm-2. We show that in ITGCN expression of p53 protein correlates with $\mathrm{p} 21$, and $\mathrm{mdm}-2$ protein is not expressed. Interestingly, we have also noted that in invasive tumors a loss of $\mathrm{p} 21$ protein is associated 
with expression of the p53 inhibitor mdm-2. In the majority of cases analyzed induction of $\mathrm{mdm}-2$ is associated with the subsequent loss of p21 expression in the invasive tumor.

\section{MATERIALS AND METHODS}

\section{Case Selection}

Germ cell tumors were identified from radical orchiectomies performed at Brigham and Women's Hospital between 1988 and 1997. Seventeen pure seminomas and 14 mixed germ cell tumors composed predominantly of embryonal carcinoma (average $75.5 \%$ ) were identified, and the diagnosis was confirmed by two independent surgical pathologists (AAR and MWD). Staging was performed using the AJCC (1997) staging system for testicular tumors. ITGCN was defined as the presence of large atypical gonocytes present adjacent to the basal wall of the seminiferous tubules. These cells have mild to moderate clear to light pink cytoplasm, enlarged nuclei, thick nuclear membranes, and prominent pink glassy nucleoli. The presence of associated displaced Sertoli cells was also noted. Recut blank slides for immunohistochemistry were composed of seminoma or embryonal carcinoma and confirmed on a hematoxylin and eosin stained section.

\section{Immunohistochemistry}

Immunohistochemistry was performed with antibodies directed against p53 (Ab-6, Calbiochem, San Diego, CA, at 1:500), p21 (Ab-1, Calbiochem, at 1:50) and mdm-2 (SMP-14, DAKO, Cupertino, CA, at 1:50). The conditions for immunohistochemistry on formalin-fixed paraffin-embedded sections has been previously described (6). In brief, antibodies were incubated on de-paraffinized sections after microwave pretreatment to expose antigen and visualized using the Vector3,3'-diaminobenzidine detection system (Vector Laboratories, Burlingame, CA) and counterstained with hematoxylin (Gill's formulation, Fisher Scientific, Springfield NJ).

\section{Scoring Methodology}

Slides were scored for tumor positivity, percentage of positive tumor cells (four independent areas of highest staining with 50 cells per area counted), intensity of staining (0-3 scale, 0 : no staining; 1 : weak staining observed over hematoxylin-counterstained chromatin; 2: moderate staining that partially obscured the counterstained chromatin; 3: strong staining that completely obscured the counterstained chromatin) and pattern of staining (nuclear, nucleolar, cytoplasmic, membranous). Negative controls (with omission of the primary antibody) and p53 and mdm-2 positive controls (bladder tumor and glioblastoma samples, respectively) were prepared in parallel with the testis tumor specimens. The presence of nuclear staining in Leydig cells (p21) was regarded as a positive internal control for that antibody. p21, p53 or mdm-2 staining was only considered positive if nuclear staining was identified within the samples. ITGCN was scored as positive or negative based on the presence or absence staining. The limited volume of ITGCN in some cases obviated the ability to determine percentages of tumor cell positivity. Statistical analysis was performed using $2 \times 2$ tables with the Fisher's exact test (two-tailed) or Mann-Whitney test (two-tailed) for grouped data. Calculations were performed with an InStat statistical evaluation program (GraphPad Software, San Diego, CA).

\section{Tumor Characteristics}

Thirty-one tumors were included in this series, 17 malignant mixed germ cell tumors with the majority component of embryonal carcinoma and 14 seminomas. The seminomas occurred at a mean age of $37+4.8$ years and embryonal carcinomas presenting at a mean age of $33+7.0$ years. Tumor size averaged $4.1 \mathrm{~cm}+1.6 \mathrm{~cm}$ (seminomas), and 3.1 $+2.2 \mathrm{~cm}$ (embryonal carcinomas). Lymphovascular invasion was present in 12 out of 31 cases (seven embryonal carcinoma and five seminoma), penetration through the tunica by tumor in 9 out of 31 cases (five embryonal carcinoma and four seminoma). Of the 14 embryonal carcinomas, three were pure embryonal carcinomas, with the remaining 11 being mixed germ cell tumors composed of an average of $75 \%$ embryonal carcinoma with secondary components including yolk sac tumor, and teratoma.

\section{Identification of Areas of ITGCN}

Each of the sections were analyzed for the presence of seminiferous tubules and when these were present for ITGCN. Examination of the sections revealed 27 cases of diagnostic ITGCN adjacent to tumors, consisting of 14 seminomas and 13 embryonal carcinomas. This represented $82 \%$ of the seminomas and $93 \%$ of the embryonal carcinoma cases. The presence of ITGCN did not correlate with size, lymphovascular invasion, tunica invasion, stage, or relapse $(P>.05)$.

\section{Results}

\section{Expression of p53 and p21 in ITGCN}

The intratubular tumors $(13 / 14$ seminomas and 13/13 embryonal carcinomas) were all positive for p53 (Table 1, Fig. 1). Eighteen out of twenty-seven (67\%) of the specimens, including 9 out of 14 sem- 
inomas (64\%) and 9 out of 13 (69\%) embryonal carcinomas, were positive for p21 in intratubular tumor. Of note, 16 of the 27 cases $(59 \%)$ revealed positive intratubular p21 expression associated with a negative p21 expression in the invasive tumor, and an additional nine cases were negative for p21 in both the invasive and intratubular tumor. Two cases were positive for p21 in both invasive and intratubular tumor. In none of the cases was the reverse (p21 positive invasive tumor and negative intratubular tumor) noted.

\section{Expression of $\mathrm{mdm}-2$ in ITGCN}

Mdm-2 was present in only two cases $(7 \%)$ of ITGCN, one embryonal carcinoma and one seminoma. The remaining 25 cases, including 13 seminomas and 12 embryonal carcinomas were negative for mdm-2 protein. When correlated with mdm-2 expression in invasive tumors (Table 1), 18 out of the ITGCN 27 cases (67\%) were positive for mdm-2 in the invasive tumor and negative for mdm-2 in the intratubular tumor. Seven of 27 (26\%) cases were negative for $\mathrm{mdm}-2$ in both the invasive tumor and ITGCN, and 2 out of 27 (7\%) were positive in both the invasive and intratubular tumor. This trend did not reach statistical significance.

Expression of p53 and p21 in Invasive Germ Cell Tumors

Thirty-one invasive tumors were stained for p53 protein by immunohistochemistry (Table 1, Fig. 1). All of the 17 seminomas and 14 of 14 embryonal carcinomas were positive for p53. Of note, embryonal carcinomas tended to have higher percentages of positive cells $(71 \pm 25 \%)$ than seminomas (25 \pm $16 \%)$, a difference that was statistically significant $(P<.0001)$. Stronger staining was also noted in embryonal carcinomas when compared with seminomas $(2.57 \pm 0.51$ versus $1.94 \pm 0.74, P=.0122)$. In contrast none of the 17 seminomas and only 2 of 14 embryonal carcinomas stained positively for $\mathrm{p} 21$ protein. Internal control Leydig cells were positive documenting the success of the p21 immunohistochemistry. Both embryonal carcinomas that were positive for p21 expressed low levels of positive cells (7 and $18 \%$ each) with staining intensity of 2 or 3

TABLE 1. Immunohistochemistry: p53, p21, and mdm-2

\begin{tabular}{|c|c|c|c|c|c|c|}
\hline & \multicolumn{3}{|c|}{ Invasive Seminoma } & \multicolumn{3}{|c|}{ Invasive Embryonal Carcinoma } \\
\hline & p53 & $\mathrm{p} 21$ & mdm-2 & p53 & $\mathrm{p} 21$ & $\mathrm{mdm}-2$ \\
\hline Number of cases & 17 & 17 & 17 & 14 & 14 & 14 \\
\hline Number positive & 17 & 0 & 8 & 14 & 2 & 13 \\
\hline Tumor positivity (\%) & 100 & 0 & 47 & 100 & 14 & 93 \\
\hline Cellular positivity (\%) & 25 & 0 & 13 & 71 & 12 & 94 \\
\hline \multirow[t]{2}{*}{ Staining intensity $(0-3)$} & 1.9 & 0.0 & 2.1 & 2.6 & 2.5 & 2.9 \\
\hline & \multicolumn{3}{|c|}{ Intratubular Seminoma } & \multicolumn{3}{|c|}{ Intratubular Embryonal Carcinoma } \\
\hline Number of Cases & 14 & 14 & 14 & 13 & 13 & 13 \\
\hline Number Positive & 13 & 9 & 1 & 13 & 9 & 1 \\
\hline Tumor Positivity (\%) & 93 & 64 & 7 & 100 & 69 & 8 \\
\hline Staining Intensity $(0-3)$ & 3.0 & 1.0 & 1.0 & 3.0 & 2.1 & 1.0 \\
\hline
\end{tabular}

\section{p53}

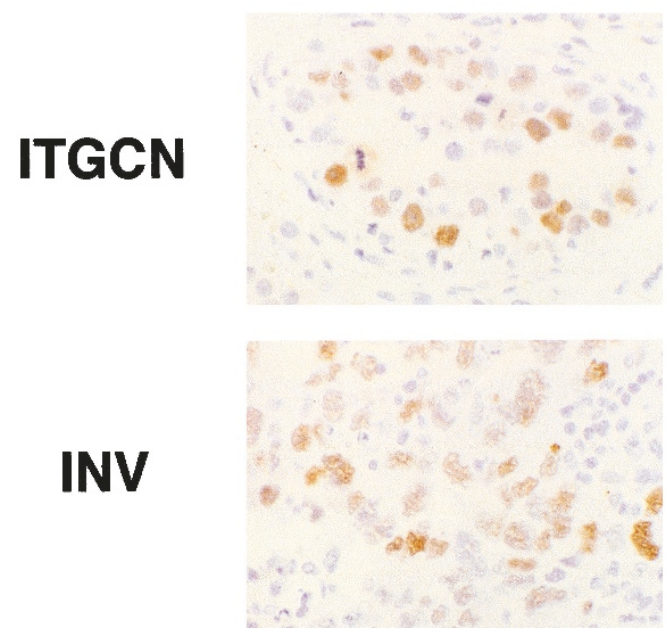

\section{mdm-2}
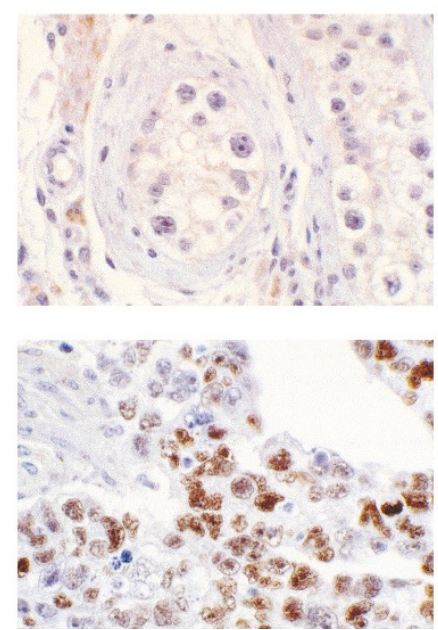

p21
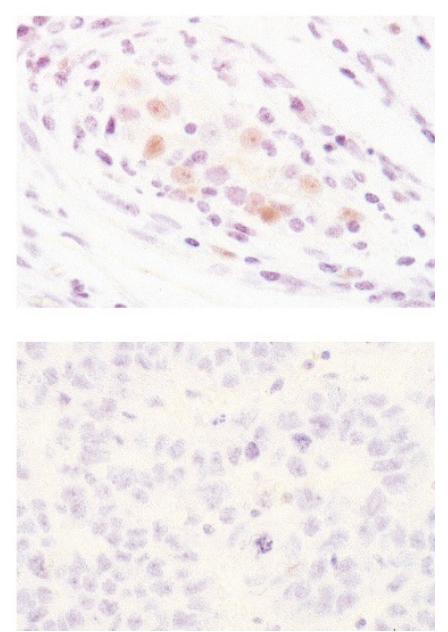

FIGURE 1. p53, mdm-2, and p21 in germ cell tumors. Invasive tumors (INV): p53, mdm-2 (400×), p21; intratubular germ cell neoplasia (ITGCN): p53, p21, mdm-2 $(400 \times)$. 
out of 3. No significant statistical correlation was noted for size, lymphovascular invasion, tunica invasion, stage, or relapse $(P>.05)$.

\section{Expression of mdm-2 in Invasive Tumors}

Mdm-2 expression by immunohistochemistry was present in 21 of 31 invasive germ cell tumors (68\%) (Table 1, Fig. 1). Only 8 of 17 (47\%) seminomas stained positively, whereas 13 of 14 (93\%) embryonal carcinomas were positive $(P=.0089)$. In the embryonal carcinomas there was stronger staining $(2.9 \pm 0.3$ versus $2.1 \pm 0.6, P=.0085)$ and greater percentages of cell staining (94 \pm 4.3 versus $13 \pm 6.2, P<.0001)$ than in the seminomas. No significant statistical correlation was noted for size, lymphovascular invasion, tunica invasion, stage, or relapse $(P>.05)$.

Thus, a pattern of ITGCN that is p53 positive, p21 positive, and mdm-2 negative, and invasive germ cell tumors that are p53 positive, p21 negative, and mdm-2 positive is present in this tumor series (Table 2).

\section{DISCUSSION}

\section{The Role of p53 in Testicular Tumors}

The presence of p53 in invasive testicular germ cell tumors is well documented (1-3). Of note, mutational analyses of p53 in testicular tumors revealed that the majority of testicular tumors overexpress wild type p53 that still maintains its ability to induce $\mathrm{p} 21$ transcription in in vitro yeast expression systems $(2,3)$. The situation in ITGCN is not as well documented. In one paper the examination of p53 in ITGCN has led to the identification of p53 mutations in a subset of microdissected cells, although a lack of p53 mutation correlation between the two methods used (DNA sequencing and singlestranded conformation polymorphism) and the identification of wild-type p53 in invasive tumors with mutant p53 in ITGCN confounds these results (7). Our results support the presence of overexpressed p53 in ITGCN. The corresponding presence of p21 expression in these cells suggests that the p53 protein may be functionally active. The identification of invasive germ cell tumors that continue to express p53 but have subsequently stopped p21

TABLE 2. Statistical Correlations: p53, p21, and mdm-2

\begin{tabular}{lccc}
\hline & $\mathrm{p} 53$ & $\mathrm{p} 21$ & $\mathrm{mdm}-2$ \\
\hline ITGCN & $26 / 27$ & $18 / 27$ & $2 / 27$ \\
Invasive & $31 / 31$ & $2 / 31$ & $21 / 31]^{*}$ \\
Seminoma & $17 / 17$ & $0 / 17$ & $8 / 17$ \\
Embryonal carcinoma & $15 / 15$ & $2 / 15$ & $13 / 15$ \\
\hline${ }^{*} P<.0001$ & \\
ITGCN, intratubular germ cell neoplasia. &
\end{tabular}

expression raises the possibility that a disruption of the p53 regulatory pathway may have occurred.

\section{The p53 Inducible Gene p21}

The induction of p53 by DNA damage from ionizing radiation or chemotherapy leads to a subsequent production of p53 response proteins. One such protein is p21 $(8,9)$, which is actively transcribed in the presence of p53 protein bound to a site in the p21 promoter region $(10,11)$. The transcription and subsequent translation of p21 leads to cell cycle arrest through p21's inhibitory interactions with the cyclins/cyclin-dependent kinases. In the normal cell this leads to a pause in cell cycle progression to allow for the repair of DNA damage. This is the first study to examine the role of p21 in ITGCN and to correlate its expression with mdm-2 and p53. In contrast to the invasive germ cell tumors, the majority of cases of ITGCN were p53 and p21 positive. This pattern contrasts with the cases of invasive tumor where p53 expression is not associated with p21 protein. Although it is possible that a disruption of the p53-p21 pathway may have occurred, other possibilities can be entertained. In vitro studies have shown that $\mathrm{p} 21$ is degraded by ubiquitin-mediated proteolysis in a p53 independent manner, a finding that could explain some reports of p53 independent control of p21 expression, which may be due to changes in cellular proteolysis. Alternatively, the expression of p21 could be mediated by other transcription factors (12). Although the inverse correlation between mdm-2 and p21 expression may suggest a role for p53 in p21 expression in the testis, it does not prove a direct cause and effect relationship. The presence of two cases where p21 expression is preserved in the invasive tumors suggests that the loss of $\mathrm{p} 21$ function may not be essential for the invasive phenotype. The fact that both of these cases also expressed $\mathrm{mdm}-2$ infers that the $\mathrm{p} 53 / \mathrm{mdm}-2$ pathway is not the only mechanism for controlling p21 protein levels in the cell. Whether these two cases represent functionally inactive mutant $\mathrm{p} 21$ proteins has not been examined, but is of interest.

\section{Regulation of $\mathrm{p} 53$ by $\mathrm{mdm}-2$}

Like p21, p53 induces the expression of mdm-2 at the level of transcription. mdm-2 protein in turn directly feeds back on p53, interacting with and inhibiting its actions. In our data, p21 expression in the presence of p53 in ITGCN is associated with a lack of mdm-2. There is a strong correlation between the onset of mdm-2 expression and the loss of p21 in the invasive tumors. Previous studies in esophageal adenocarcinomas have shown a similar correlation between wild type p53 expression and 
mdm-2 expression, and have argued that mdm-2/ p53 expression abrogates the tumor-suppressor phenotype of p53 (13). This expression of mdm-2 has been associated with amplification of the mdm-2 gene. Similarly in gliomas, another type of tumor that has been shown to express high levels of wild-type p53, low levels of p21 are correlated with high levels of mdm-2, supporting a model of mdm-2 blocking the p53 dependent activation of p21 (14). Mutant forms of mdm-2 have been shown to stabily inactivate p53 without inducing p53 degradation (15). Thus mdm-2 mutations could explain the high levels of mdm-2 and wild type p53 in testicular tumors that lack p21 expression, a proposal that is currently under investigation. Although intriguing, this model does not explain the lack of mdm-2 in 9 of 17 invasive seminomas, all of which did not express $\mathrm{p} 21$. These cases suggest that alternate regulatory pathways may be involved in the downregulation of $\mathrm{p} 21$, possibly including proteins such as mdm-x, a newly described mdm-2like protein that also inhibits p53 function (16). Examination of the actual mechanism of p21 regulation in testis tumors is required before a definitive model can be proven.

\section{Loss of p21 Expression is Associated with the Development of Invasive Germ Cell Tumors}

In the cases that have been analyzed the major pattern that has been seen are p53+/p21+ intratubular tumor cells. The subsequent progression to a p53+/p21 - invasive phenotype is inferred by the presence of such tumor cells in the associated invasive tumor. Also supporting this model is the presence of smaller numbers of p53+/p21 - intratubular cells as a possible transitional state in invasive tumorigenesis, although the possibility that these cells represent pagetoid spread of an invasive germ cell tumor cannot be excluded. The ability to examine p21 expression in cases of pure ITGCN without an associated invasive germ cell tumor might allow one to further delineate the role of $\mathrm{p} 21$ in invasion, although one cannot definitively prove that the cells examined do not represent early intratubular spread of an invasive tumor. The complete lack of p53-/p21+ tumor cells lends support to the model whereby control of p21 expression may be through p53, and p53 independent induction has not occurred.

Based on these findings, a theoretical model of invasive germ cell tumor formation can be proposed that would include the induction of p53 followed by the subsequent loss of p53 regulated p21 induction through the expression of mdm-2 (Fig. 2). This loss of p21 cell cycle inhibition may lead to the loss of growth control that is crucial to neoplastic transformation. The loss of p21 activity, coupled with our previous
A

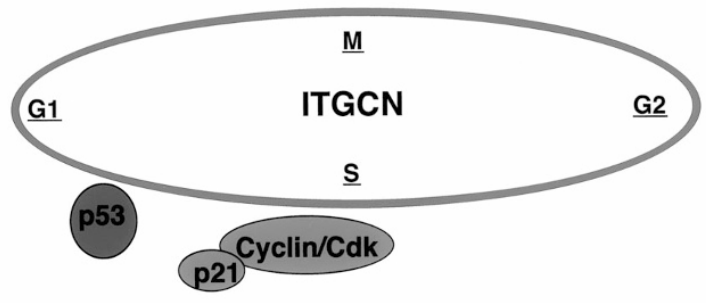

B

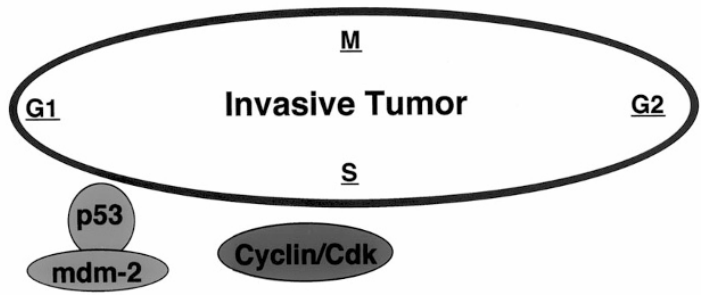

FIGURE 2. A proposed model for $\mathrm{p} 53 / \mathrm{p} 21 / \mathrm{mdm}-2$ regulation in germ cell tumors. A, Intratubular germ cell neoplasia (ITGCN): p53 induces p21 in the absence of mdm-2. p21 binds and inactivates cyclin/cdk complexes and inhibits their activity, resulting in cell cycle arrest. B, Invasive tumor: mdm-2 binds p53 and blocks induction of p21, allowing for cyclin/cdk activity and continued cellular proliferation.

data showing that cyclin A and cyclin E are overexpressed in germ cell tumors (17) leads to a model of uncontrolled cellular proliferation in testis tumors. Although only theoretical, this model can be tested using in vitro and in vivo experimental systems. The loss of p21 protein through the functional inhibition of p53 by mdm-2 is currently being evaluated. An association between the loss of p21 expression and development of the invasive phenotype would lead to questions regarding the role of cellular proliferation in tumor invasion.

\section{REFERENCES}

1. Riou G, Barrois M, Prost S, Terrier MJ, Theodore C, Levine AJ. The p53 and mdm-2 genes in human testicular germ-cell tumors. Mol Carcinog 1995;12:124-31.

2. Guillou L, Estreicher A, Chaubert P, Hurlimann J, Kurt AM, Metthez G, et al. Germ cell tumors of the testis overexpress wild-type p53. Am J Pathol 1996;149:1221-8.

3. Schenkman NS, Sesterhenn IA, Washington L, Tong YA, Weghorst CM, Buzard GS, et al. Increased p53 protein does not correlate to p53 gene mutations in microdissected human testicular germ cell tumors. J Urol 1995;154:617-21.

4. Gartel AL, Serfas MS, Tyner AL. p21-negative regulator of the cell cycle. Proc Soc Exp Biol Med 1996;213:138-49.

5. Haupt Y, Maya R, Kazaz A, Oren M. Mdm2 promotes the rapid degradation of p53. Nature 1997;387(6630):296-9.

6. Renshaw AA, Loughlin KR, Dutta A. Cyclin A and MIB1 (Ki67) as markers of proliferative activity in primary renal neoplasms. Mod Pathol 1998;11:963-6.

7. Kuczyk MA, Serth J, Bokemeyer C, Jonassen J, Machtens S, Werner M, et al. Alterations of the p53 tumor suppressor gene in carcinoma in situ of the testis. Cancer 1996;78:195866.

8. Wu L, Levine AJ. Differential regulation of the p21/WAF-1 and mdm2 genes after high-dose UV irradiation: p53- 
dependent and p53-independent regulation of the mdm2 gene. Mol Med 1997;3:441-51.

9. Momand J, Zambetti GP. Mdm-2: 'big brother' of p53. J Cell Biochem 1997;64:343-52.

10. Tang HY, Zhao K, Pizzolato JF, Fonarev M, Langer JC, Manfredi JJ. Constitutive expression of the cyclin-dependent kinase inhibitor p21 is transcriptionally regulated by the tumor suppressor protein p53. J Biol Chem 1998;273:29156-63.

11. Chin PL, Momand J, Pfeifer GP. In vivo evidence for binding of p53 to consensus binding sites in the p21 and GADD45 genes in response to ionizing radiation. Oncogene 1997;15: 87-99.

12. Gartel AL, Tyner AL. Transcriptional regulation of the p21((WAF1/CIP1)) gene. Exp Cell Res 1999;246:280-9.

13. Soslow RA, Altorki NK, Yang G, Xie D, Yang CS. mdm-2 expression correlates with wild-type p53 status in esopha- geal adenocarcinoma. Mod Pathol 1999;12:580-6.

14. Pykett MJ, Azzam E, Dahlberg W, Little JB. Differential p53, $\mathrm{p} 21, \mathrm{mdm} 2$ and $\mathrm{Rb}$ regulation in glioma cell lines that overexpress wild-type p53. Int J Oncol 1998;13:213-6.

15. Kubbutat MH, Ludwig RL, Levine AJ, Vousden KH. Analysis of the degradation function of Mdm2. Cell Growth Differ 1999;10:87-92.

16. Shvarts A, Bazuine M, Dekker P, Ramos YF, Steegenga WT, Merckx G, et al. Isolation and identification of the human homolog of a new p53-binding protein, Mdmx. Oncogene 1999;18:189-99.

17. Datta MW, Renshaw AA, Dutta A, Hoffman MA, Loughlin KR. Evaluation of cyclin expression in testicular germ cell tumors: cyclin E correlates with tumor type, advanced clinical stage, and pulmonary metastasis. Mod Pathol 2000;13:66772 . 